\title{
Discontinuous dynamic equations on time scales
}

\author{
Iguer Luis Domini dos Santos ${ }^{1}$
}

Received: 11 March 2015 / Accepted: 20 May 2015 / Published online: 27 May 2015

(C) Springer-Verlag Italia 2015

\begin{abstract}
We introduce and prove the existence of Hermes, Filippov, and Krasovskii generalized solutions to discontinuous dynamic equations on time scales. We also consider comparisons between the Carathéodory, Euler, Filippov, Hermes, and Krasovskii generalized solutions to discontinuous dynamic equations on time scales.
\end{abstract}

Keywords Generalized solutions · Initial value problems · Dynamic equations ·

Time scales

Mathematics Subject Classification $34 \mathrm{~A} 12 \cdot 34 \mathrm{~A} 36 \cdot 34 \mathrm{~N} 05$

\section{Introduction}

In this report we introduce three concepts of generalized solutions to discontinuous dynamic equations on time scales. These three concepts are those involved in the Hermes, Filippov, and Krasovskii solutions. Such generalized solutions to dynamic equations on time scales are extensions of the concepts of generalized solutions to discontinuous differential equations $[11,15,17,18]$.

The study of dynamic equations of the first order on time scales can be found, for example, in $[3,7,10,12,20,23,26]$. In particular, Euler solutions were introduced in [23] while Carathéodory solutions were treated in [7,12].

We extend to systems of dynamic equations on time scales results of the existence of solutions to discontinuous differential equations, see, for example $[6,11,15,16]$. Thus, we prove the existence of Carathéodory, Hermes, Filippov, and Krasovskii solutions to discontinuous dynamic equations on time scales. The existence of Carathéodory solutions were obtained without the assumption that the vector field of the dynamic equation be bounded. To the

Iguer Luis Domini dos Santos iguerluis@mat.feis.unesp.br

1 Departamento de Matemática, Faculdade de Engenharia de Ilha Solteira, UNESP, Univ Estadual Paulista, Rua Rio de Janeiro, 266, Ilha Solteira, São Paulo CEP 15385-000, Brazil 
best of our knowledge, the results of the existence of solutions for discontinuous dynamic equations on time scales obtained in this report have not been considered in the literature.

We also studied comparisons between Carathéodory, Euler, Hermes, Filippov, and Krasovskii solutions to dynamic equations on time scales. Among these comparisons, we found that some properties of solutions to discontinuous differential equations $[15,17]$ are not valid to discontinuous dynamic equations on time scales.

\section{Background and preliminaries}

In this section, we consider basic concepts and results that will be used throughout the work.

We will use the following conventions:

(i) if $x \in \mathbb{R}^{n}$ we denote the Euclidean norm of $x$ by $\|x\|$;

(ii) $B$ is the open unit ball $\left\{x \in \mathbb{R}^{n}:\|x\|<1\right\}$;

(iii) for $M \subset \mathbb{R}^{n}, \overline{c o} M$ denotes closure of the convex hull of $\mathrm{M}$;

(iv) given a compact subset $E \subset \mathbb{R}$ and a function $g: E \rightarrow \mathbb{R}^{n}$, we will denote by $\|g\|_{\infty}$ the supremum norm.

\subsection{Calculus on time scales}

A time scale is a nonempty closed subset $\mathbb{T} \subset \mathbb{R}$ of the real numbers. We will use an arbitrary bounded time scale $\mathbb{T}$ such that $a=\min \mathbb{T}$ and $b=\max \mathbb{T}$. Furthermore, we suppose that $a<b$.

We define the forward jump operator $\sigma: \mathbb{T} \rightarrow \mathbb{T}$ by

$$
\sigma(t)=\inf \{s \in \mathbb{T}: s>t\}
$$

and the backward jump operator $\rho: \mathbb{T} \rightarrow \mathbb{T}$ by

$$
\rho(t)=\sup \{s \in \mathbb{T}: s<t\} .
$$

Here we assume that inf $\emptyset=\sup \mathbb{T}$ and $\sup \emptyset=\inf \mathbb{T}$.

Lemma 1 [5] There exist $I \subset \mathbb{N}$ and $\left\{t_{i}\right\}_{i \in I} \subset \mathbb{T}$ such that

$$
R S:=\{t \in \mathbb{T}: t<\sigma(t)\}=\left\{t_{i}\right\}_{i \in I},
$$

where $R S$ stands for right scattered points of the time scale $\mathbb{T}$.

Define the function $\mu: \mathbb{T} \rightarrow[0,+\infty)$ by

$$
\mu(t)=\sigma(t)-t .
$$

If $A \subset \mathbb{R}$, we define the set $A_{\mathbb{T}}$ by $A_{\mathbb{T}}=A \cap \mathbb{T}$. We define $\mathbb{T}^{\kappa}:=\mathbb{T} \backslash(\rho(\sup \mathbb{T}) \text {, sup } \mathbb{T}]_{\mathbb{T}}$.

Consider a function $f: \mathbb{T} \rightarrow \mathbb{R}$ and $t \in \mathbb{T}^{\kappa}$. If $\xi \in \mathbb{R}$ is such that, for all $\varepsilon>0$ there exists $\delta>0$ obeying

$$
|f(\sigma(t))-f(s)-\xi(\sigma(t)-s)| \leq \varepsilon|\sigma(t)-s|
$$

for all $s \in(t-\delta, t+\delta)_{\mathbb{T}}$, we say that $\xi$ is the delta derivative of $f$ at $t$ and we denote it by $\xi:=f^{\Delta}(t)$.

Now, consider a function $f: \mathbb{T} \rightarrow \mathbb{R}^{n}$ and $t \in \mathbb{T}^{\kappa}$. We say that $f$ is $\Delta$-differentiable at $t$ if each component $f_{i}: \mathbb{T} \rightarrow \mathbb{R}$ of $f$ is $\Delta$-differentiable at $t$. In this case $f^{\Delta}(t)=$ $\left(f_{1}^{\Delta}(t), \ldots, f_{n}^{\Delta}(t)\right)$. 
The following result is proven in [2] for scalar valued functions. But the generalization for vector valued functions is straightforward.

Theorem 1 [2] Consider a function $f: \mathbb{T} \rightarrow \mathbb{R}^{n}$ and $t \in \mathbb{T}^{\kappa}$. Then the following statements hold:

(i) If $f$ is $\Delta$-differentiable at $t$ then $f$ is continuous at $t$.

(ii) If $f$ is continuous at $t$ and $\sigma(t)>t$, then $f$ is $\Delta$-differentiable at $t$. Furthermore,

$$
f^{\Delta}(t)=\frac{f(\sigma(t))-f(t)}{\mu(t)} .
$$

(iii) If $\sigma(t)=t$, then $f$ is $\Delta$-differentiable at $t$ if and only if there exists the limit

$$
\lim _{s \rightarrow t} \frac{f(t)-f(s)}{t-s}
$$

as an element of $\mathbb{R}^{n}$. In that case

$$
f^{\Delta}(t)=\lim _{s \rightarrow t} \frac{f(t)-f(s)}{t-s} .
$$

(iv) If $f$ is $\Delta$-differentiable at $t$, then

$$
f(\sigma(t))=f(t)+\mu(t) f^{\Delta}(t) .
$$

Consider a nonempty compact subset $K \subset \mathbb{R}$. A partition of $K$ is a set $P=$ $\left\{t_{0}, t_{1}, \ldots, t_{N}\right\} \subset K$ such that $t_{0}=c<t_{1}<\cdots<t_{N}=d$, where $c=\min K$ and $d=\max K$. For a given $\delta>0$, we denote by $\mathcal{P}_{\delta}(K)$ the set of all partitions $P=\left\{t_{0}, t_{1}, \ldots, t_{N}\right\}$ of $K$ satisfying:

(i) if $t_{i}-t_{i-1}>\delta$ for some $i$ then $\rho\left(t_{i}\right)=t_{i-1}$; or

(ii) $t_{i}-t_{i-1} \leq \delta$ otherwise.

Lemma 2 [14] For each $\delta>0$ the set $\mathcal{P}_{\delta}(K)$ is nonempty.

\section{2 $\Delta$-measurable sets}

Below, we recall the $\sigma$-algebra of subsets of the time scale $\mathbb{T}$.

Denote by $\mathfrak{F}$ the collection of all subintervals of $\mathbb{T}$ of the form $[\tilde{a}, \tilde{b})_{\mathbb{T}}=\{t \in \mathbb{T}: \tilde{a} \leq$ $t<\tilde{b}\}$, where $\tilde{a}, \tilde{b} \in \mathbb{T}$. The interval $[\tilde{a}, \tilde{a})_{\mathbb{T}}$ is understood as an empty set.

Let $E$ be an arbitrary subset of $\mathbb{T}$. If there exists at least one sequence of intervals $\left[a_{j}, b_{j}\right)_{\mathbb{T}} \in \mathfrak{F}$ such that $E \subset \bigcup_{j}\left[a_{j}, b_{j}\right)_{\mathbb{T}}$, the outer measure of $E$ is defined by

$$
m^{*}(E)=\inf \left\{\sum_{k=1}^{+\infty}\left(b_{k}-a_{k}\right): E \subset \bigcup_{k}\left[a_{k}, b_{k}\right)_{\mathbb{T}},\left[a_{k}, b_{k}\right)_{\mathbb{T}} \in \mathfrak{F}\right\} .
$$

If there is no such a cover of $E$ we set $m^{*}(E)=+\infty$.

The outer measure defined on $\mathbb{R}$ will be denoted by $\lambda^{*}$.

Properties of the outer measure $m^{*}$ defined previously can be found in $[5,13,21]$. Below, we have presented some of these properties.

Lemma 3 [13] If $c, d \in \mathbb{T}$ and $c<d$ then

$$
m^{*}\left([c, d)_{\mathbb{T}}\right)=d-c .
$$

By [21] one can prove the following lemma. 
Lemma 4 [21] If $\left\{E_{i}\right\}_{i \in \mathbb{N}}$ is a sequence of subsets of $\mathbb{T}$, then

$$
m^{*}\left(\bigcup_{i=1}^{+\infty} E_{i}\right) \leq \sum_{i=1}^{+\infty} m^{*}\left(E_{i}\right) .
$$

Lemma 5 [5] Let $E \subset[a, b)_{\mathbb{T}}$ be such that $E \subset\{t \in \mathbb{T}: \sigma(t)=t\}$. Then

$$
m^{*}(E)=\lambda^{*}(E) \text {. }
$$

Definition 1 A set $E \subset \mathbb{T}$ is said to be $\Delta$-measurable (Lebesgue $\Delta$-measurable) if

$$
m^{*}(A)=m^{*}(A \cap E)+m^{*}(A \cap(\mathbb{T} \backslash E))
$$

for each $A \subset \mathbb{T}$.

Proposition 1 [5] Take $E \subset \mathbb{T}$. Then $E$ is $\Delta$-measurable if and only if $E$ is Lebesgue measurable.

As a consequence we have the following result.

Theorem 2 The family of $\Delta$-measurable sets is a $\sigma$-algebra on $\mathbb{T}$.

Denote by $\Delta$ the $\sigma$-algebra of $\Delta$-measurable subsets of $\mathbb{T}$. The measure $m^{*}: \Delta \rightarrow$ $[0,+\infty]$ will be called $\Delta$-measure of Lebesgue and it is denoted by $m^{*} \equiv \mu_{\Delta}$.

Let $E \subset \mathbb{T}$. We say that a statement $P$ holds $\Delta$-almost everywhere ( $\Delta$-a.e.) on $E$, if the set $N$ given by

$$
N=\{t \in E: P \quad \text { does not hold at } t\}
$$

satisfies $\mu_{\Delta}(N)=0$.

\section{$2.3 \Delta$-measurable functions and $\Delta$-integrability}

It is said that a function $f: \mathbb{T} \rightarrow[-\infty,+\infty]$ is $\Delta$-measurable if for each $r \in \mathbb{R}$ the set $\{t \in \mathbb{T}: f(t)<r\}$ is $\Delta$-measurable. A vector valued function $f: \mathbb{T} \rightarrow \mathbb{R}^{n}$ is called $\Delta$-measurable if each component $f_{i}: \mathbb{T} \rightarrow \mathbb{R}$ of $f$ is $\Delta$-measurable.

Given a function $f: \mathbb{T} \rightarrow \mathbb{R}^{n}$ we define $\tilde{f}:[a, b] \rightarrow \mathbb{R}^{n}$ by

$$
\tilde{f}(t)=\left\{\begin{array}{l}
f(t), \quad t \in \mathbb{T} \\
f\left(t_{i}\right), \quad t \in\left(t_{i}, \sigma\left(t_{i}\right)\right) \quad \text { for some } i \in I
\end{array}\right.
$$

where $I \subset \mathbb{N}$ and $\left\{t_{i}\right\}_{i \in I} \subset \mathbb{T}$ are such that $\{t \in \mathbb{T}: t<\sigma(t)\}=\left\{t_{i}\right\}_{i \in I}$.

Proposition 2 [5] Consider a function $f: \mathbb{T} \rightarrow \mathbb{R}^{n}$. Then $f$ is $\Delta$-measurable if and only if $\tilde{f}$ is Lebesgue measurable.

Proof This result is an direct consequence of [5, Proposition 4.1].

The Lebesgue integral of a function $f: \mathbb{T} \rightarrow \overline{\mathbb{R}}$ with respect to the measure $\mu_{\Delta}$ over a set $E \in \Delta$ is denoted by

$$
\int_{E} f(s) \Delta s
$$

We call this integral the Lebesgue $\Delta$-integral of $f$ over $E$ and denote the set of functions $f: \mathbb{T} \rightarrow \mathbb{R}$ which are $\Delta$-integrable over $E$ by $L_{1}(E)$. When $f: \mathbb{T} \rightarrow \mathbb{R}^{n}$ is a $\Delta$-measurable 
function and $E \in \Delta, f$ is integrable over $E$ if each component $f_{i}: \mathbb{T} \rightarrow \mathbb{R}$ is integrable over $E$. We also denote by $L_{1}\left(E, \mathbb{R}^{n}\right)$ the set of functions $f: \mathbb{T} \rightarrow \mathbb{R}^{n} \Delta$-integrable over $E$.

Next, we consider a result that relates the Lebesgue $\Delta$-integral on time scales and usual Lebesgue integral and that is needed in the sequel.

If $E \subset \mathbb{T}$ define the set $\tilde{E}$ as

$$
\tilde{E}=E \cup \bigcup_{i \in I_{E}}\left(t_{i}, \sigma\left(t_{i}\right)\right)
$$

where

$$
I_{E}:=\left\{i \in I: t_{i} \in E \cap R S\right\} .
$$

The following result is proven in [5] for scalar valued functions. However, one can readily see that it holds for vector valued functions as stated below.

Theorem 3 [5] Take a $\Delta$-measurable set $E \subset \mathbb{T}$ such that $b \notin E$. Let $f: \mathbb{T} \rightarrow \mathbb{R}^{n}$ be $a$ $\Delta$-measurable function and $\tilde{f}:[a, b] \rightarrow \mathbb{R}^{n}$ the extension of $f$ previously defined. Then $f \in L_{1}\left(E, \mathbb{R}^{n}\right)$ if and only if $\tilde{f} \in L_{1}\left(\tilde{E}, \mathbb{R}^{n}\right)$. In this case

$$
\int_{E} f(s) \Delta s=\int_{\tilde{E}} \tilde{f}(s) d s .
$$

\subsection{Absolutely continuous functions on time scales}

A function $f: \mathbb{T} \rightarrow \mathbb{R}^{n}$ is absolutely continuous if given $\varepsilon>0$ there exists $\delta>0$ such that

$$
\sum_{i=1}^{N}\left\|f\left(b_{i}\right)-f\left(a_{i}\right)\right\|<\varepsilon
$$

whenever $a_{i} \leq b_{i}$ and $\left\{\left[a_{i}, b_{i}\right)_{\mathbb{T}}\right\}_{i=1}^{N}$ are disjoint intervals obeying

$$
\sum_{i=1}^{N}\left(b_{i}-a_{i}\right)<\delta .
$$

Theorem 4 given below is stated and proven in [4] for scalar valued functions. However, it is easy to check that it can be extended for vector valued functions as stated below.

Theorem 4 [4] A function $f: \mathbb{T} \rightarrow \mathbb{R}^{n}$ is absolutely continuous if and only if the following assertions are valid:

(i) for $\Delta$-a.e. $t \in[a, b)_{\mathbb{T}}$ the function $f$ is $\Delta$-differentiable and $f^{\Delta} \in L_{1}\left([a, b)_{\mathbb{T}}, \mathbb{R}^{n}\right)$;

(ii) for each $t \in \mathbb{T}$ we have

$$
f(t)=f(a)+\int_{[a, t)_{\mathbb{T}}} f^{\Delta}(s) \Delta s .
$$

A function $f: \mathbb{T} \rightarrow \mathbb{R}^{n}$ is called an arc if it is absolutely continuous.

We will use the following results concerning absolutely continuous functions on time scales.

Proposition 3 [23] Consider a sequence $\left\{x_{i}\right\}_{i \in \mathbb{N}}$ of arcs $x_{i}: \mathbb{T} \rightarrow \mathbb{R}^{n}$. Let $\phi: \mathbb{T} \rightarrow[0,+\infty)$ be in $L_{1}\left([a, b)_{\mathbb{T}}\right)$ such that for each $i$, we get

$$
\left\|x_{i}^{\Delta}(s)\right\| \leq \phi(s) \quad \Delta \text {-a.e. } s \in[a, b)_{\mathbb{T}} .
$$


If $\left\{x_{i}(a)\right\}$ is a bounded sequence, then there exists a subsequence $\left\{x_{i_{k}}\right\} \subset\left\{x_{i}\right\}$ and an arc $x: \mathbb{T} \rightarrow \mathbb{R}^{n}$ such that $x_{i_{k}} \rightrightarrows x$. Furthermore, if $c, d \in \mathbb{T}$ and $c<d$ then

$$
\lim _{k \rightarrow \infty} \int_{[c, d)_{\mathbb{T}}} x_{i_{k}}^{\Delta}(s) \Delta s=\int_{[c, d)_{\mathbb{T}}} x^{\Delta}(s) \Delta s .
$$

The notation $x_{i_{k}} \rightrightarrows x$ means uniform convergence. We also note that the above proposition remains valid if the condition given by Eq. (1) is replaced by

$$
\left\|\int_{[c, d)_{\mathbb{T}}} x_{i}^{\Delta}(s) \Delta s\right\| \leq \int_{[c, d)_{\mathbb{T}}} \phi(s) \Delta s
$$

for each $c, d \in \mathbb{T}$ such that $c<d$.

Proposition 4 [23] Let $f: \mathbb{T} \rightarrow[0,+\infty)$ be a $L_{1}\left([a, b)_{\mathbb{T}}\right)$ function. Given $\varepsilon>0$ there exists $\delta>0$ such that, if $A \in \Delta$ and $\mu_{\Delta}(A)<\delta$ then

$$
\int_{A} f(s) \Delta s<\varepsilon
$$

Lemma 6 Consider an arc $x: \mathbb{T} \rightarrow \mathbb{R}^{n}$ and a function $f \in L_{1}\left([a, b)_{\mathbb{T}}, \mathbb{R}^{n}\right)$. Let $c$ and $d$ in $\mathbb{T}$ be such that $c<d$. If, for each $t \in[c, d]_{\mathbb{T}}$, we have

$$
x(t)=\int_{[c, t)_{\mathbb{T}}} f(s) \Delta s
$$

then

$$
x^{\Delta}(s)=f(s) \quad \Delta \text {-a.e. } s \in[c, d)_{\mathbb{T}} .
$$

Proof Consider $t \in[c, d)_{\mathbb{T}}$ such that there exists the delta derivative $x^{\Delta}(t)$. If $\sigma(t)>t$ it follows that

$$
\begin{aligned}
x^{\Delta}(t) & =\frac{\int_{[c, \sigma(t))_{\mathbb{T}}} f(s) \Delta s-\int_{[c, t)_{\mathbb{T}}} f(s) \Delta s}{\mu(t)} \\
& =\frac{\int_{[t, \sigma(t))_{\mathbb{T}}} f(s) \Delta s}{\mu(t)}=f(t) .
\end{aligned}
$$

If $\sigma(t)=t$ and $t$ is a Lebesgue point of $\tilde{f}$, let $\left\{t_{i}\right\}_{i \in \mathbb{N}} \subset[c, d)_{\mathbb{T}}$ be a sequence such that $t_{i} \downarrow t$. Using Theorem 3 and the definition of a Lebesgue point [22], we obtain

$$
\begin{aligned}
x^{\Delta}(t) & =\lim _{i \rightarrow \infty} \frac{x\left(t_{i}\right)-x(t)}{t_{i}-t}=\lim _{i \rightarrow \infty} \frac{\int_{\left[t, t_{i}\right)_{\mathbb{T}}} f(s) \Delta s}{t_{i}-t} \\
& =\lim _{i \rightarrow \infty} \frac{\int_{\left[t, t_{i}\right)} \tilde{f}(s) d s}{t_{i}-t}=\tilde{f}(t)=f(t) .
\end{aligned}
$$

Thus, if $D=\left\{t \in[c, d)_{\mathbb{T}}: x^{\Delta}(t) \neq f(t)\right\}$ then $D \subset A \cap E$, where

$$
A=\left\{t \in[c, d)_{\mathbb{T}}: \sigma(t)=t\right\}
$$

and $E$ is the set of points $t \in[c, d)_{\mathbb{T}}$ such that $t$ is not a Lebesgue point of $\tilde{f}$. From Lemma 5 , we find that

$$
m^{*}(D) \leq m^{*}(A \cap E)=\lambda^{*}(A \cap E) \leq \lambda^{*}(E)=0
$$

and therefore $x^{\Delta}(t)=f(t) \Delta$-a.e. $t \in[c, d)_{\mathbb{T}}$. 


\subsection{Aumann integral}

Given a set-valued mapping $\Gamma:[a, b] \rightsquigarrow \mathbb{R}^{n}$, the Aumann integral $\int_{[a, b]} \Gamma(t) d t$ is the set consisting of all $\int_{[a, b]} \gamma(t) d t$ for Lebesgue integrable $\gamma($.$) such that \gamma(t) \in \Gamma(t)$ for each $t \in[a, b]$.

The following two results stated and proven in [15], for $a=0$ and $b=1$, will be required for obtaining the closure theorem for Krasovskii solutions.

Lemma 7 [15] Let $\Gamma:[a, b] \rightsquigarrow \mathbb{R}^{n}$ be a set-valued mapping whose values $\Gamma(t)$ are compact, convex subsets of $\mathbb{R}^{n}$, all contained in a common ball. Then $\int_{[a, b]} \Gamma(t) d t$ is compact and convex.

Lemma 8 [15] Let $\Gamma:[a, b] \rightsquigarrow \mathbb{R}^{n}$ be a set-valued mapping, whose values are all contained in a common ball of $\mathbb{R}^{n}$. If $x:[a, b] \rightarrow \mathbb{R}^{n}$ is such that

$$
x(t)-x(s) \in \int_{[s, t]} \Gamma(\tau) d \tau
$$

for all $t>s$ in $[a, b]$, then $x($.$) is an arc and satisfies$

$$
\dot{x}(\tau) \in \overline{c o} \Gamma(\tau) \quad \text { a.e. } \tau \in[a, b)
$$

In particular,

$$
x(t)=x(a)+\int_{[a, t]} \dot{x}(\tau) d \tau \in x(a)+\int_{[a, t]} \overline{c o} \Gamma(\tau) d \tau
$$

for all $t \in[a, b]$.

\section{Generalized solutions to discontinuous dynamic equations}

Consider the initial value problem

$$
\left\{\begin{array}{l}
x^{\Delta}(t)=f(t, x(t)) \quad \Delta \text {-a.e. } t \in[a, b)_{\mathbb{T}} \\
x(a)=x_{0}
\end{array}\right.
$$

where $x_{0} \in \mathbb{R}^{n}$ and $f: \mathbb{T} \times \mathbb{R}^{n} \rightarrow \mathbb{R}^{n}$. In this section, we introduce and prove the existence of Hermes, Filippov, and Krasovskii solutions to the problem stated in Eq. (2). We also prove the existence of Carathéodory solutions to Eq. (2) using the methodology already introduced for discontinuous differential equations, see, for example $[9,16]$. We extend to systems of dynamic equations on time scales the relationship $\mathcal{H} \subset \mathcal{K}$ [15, Corollary 4.4], that is, each Hermes solution is a Krasovskii solution. In addition, we remind Euler solutions to Eq. (2) and we consider comparisons between the solutions generalized throughout the section.

We denote the sets of Euler, Hermes, Filippov, and Krasovskii solutions to Eq. (2) respectively by $\mathcal{E}, \mathcal{H}, \mathcal{F}$, and $\mathcal{K}$.

We say that the function $f: \mathbb{T} \times \mathbb{R}^{n} \rightarrow \mathbb{R}^{n}$ is continuous in $x$ if for each $t \in \mathbb{T}$, the function $x \mapsto f(t, x)$ is continuous. The function $f$ is said to be $\Delta$-measurable in $t$ if and only if $t \mapsto f(t, x)$ is $\Delta$-measurable for each $x \in \mathbb{R}^{n}$. Furthermore, we say that $f$ is locally bounded, if $f$ is bounded on each bounded subset of $\mathbb{T} \times \mathbb{R}^{n}$. 


\subsection{Carathéodory solutions}

In Theorem 5 below, we prove the existence of Carathéodory solutions to Eq. (2). Unlike [7], we do not require that the vector field of the dynamic equation be bounded. Furthermore, we do not use the concept of the solution tube [12] to obtain Carathéodory solutions to Eq. (2).

Definition 2 An $\operatorname{arc} x: \mathbb{T} \rightarrow \mathbb{R}^{n}$ is a Carathéodory solution (or $\mathcal{C}$-solution) to Eq. (2) if and only if it satisfies the dynamic equation given by Eq. (2) for $\Delta$-a.e. $t \in[a, b)_{\mathbb{T}}$ and $x(a)=x_{0}$.

Consider a function $\phi: \mathbb{T} \times \mathbb{R}^{m} \rightarrow \mathbb{R}^{n}$. We say that $\phi$ is a $\Delta$-Carathéodory function if it satisfies the following properties:

(i) for each $t \in \mathbb{T}$, the function $x \mapsto \phi(t, x)$ is continuous.

(ii) for each $x \in \mathbb{R}^{m}$, the function $t \mapsto \phi(t, x)$ is $\Delta$-measurable.

We will use the following basic result.

Lemma 9 Let $\phi: \mathbb{T} \times \mathbb{R}^{m} \rightarrow \mathbb{R}^{n}$ be a $\Delta$-Carathéodory function and $x: \mathbb{T} \rightarrow \mathbb{R}^{n}$ a $\Delta$-measurable function. Then the function

$$
t \mapsto \phi(t, x(t))
$$

is $\Delta$-measurable.

Proof Define the function $\tilde{\phi}:[a, b] \times \mathbb{R}^{m} \rightarrow \mathbb{R}^{n}$ by

$$
\tilde{\phi}(t, u)=\left\{\begin{array}{l}
\phi(t, u), t \in \mathbb{T} \\
\phi\left(t_{i}, u\right), \quad t \in\left(t_{i}, \sigma\left(t_{i}\right)\right) \text { for some } i \in I .
\end{array}\right.
$$

Since $\tilde{\phi}$ is a $\mathcal{L}$-Carathéodory function [24, Lemma 4.7], it follows from [19, 2B.5. Lemma], that the function $t \mapsto \tilde{\phi}(t, \tilde{x}(t))$ is Lebesgue measurable. Thus, by Proposition 2, the function $t \mapsto \phi(t, x(t))$ is $\Delta$-measurable.

Theorem 5 (Existence for $\mathcal{C}$-solutions) Let $f$ be a $\Delta$-Carathéodory function. Suppose also that, for each $\beta>0$ there is a function $m_{\beta}: \mathbb{T} \rightarrow[0,+\infty)$ in $L_{1}\left([a, b)_{\mathbb{T}}\right)$ such that

$$
\|f(t, x)\| \leq m_{\beta}(t)
$$

whenever $t \in \mathbb{T}$ and $\|x\| \leq \beta+\left\|x_{0}\right\|$. Then there exists $b_{1} \in \mathbb{T} \backslash\{a\}$ such that the initial value problem

$$
\left\{\begin{array}{l}
x^{\Delta}(t)=f(t, x(t)) \quad \Delta \text {-a.e. } t \in\left[a, b_{1}\right)_{\mathbb{T}} \\
x(a)=x_{0}
\end{array}\right.
$$

has a Carathéodory solution.

Proof If $\sigma(a)>a$ take $b_{1}=\sigma(a)$. Define the $\operatorname{arc} x:\left[a, b_{1}\right]_{\mathbb{T}} \rightarrow \mathbb{R}^{n}$ by $x(a)=x_{0}$ and $x\left(b_{1}\right)=\mu(a) f(a, x(a))+x(a)$. Hence,

$$
x^{\Delta}(a)=\frac{x(\sigma(a))-x(a)}{\mu(a)}=f(a, x(a))
$$

and then

$$
x^{\Delta}(t)=f(t, x(t))
$$

whenever $t \in\left[a, b_{1}\right)_{\mathbb{T}}=\{a\}$. Therefore $x$ is a Carathéodory solution to Eq. (3). 
If $\sigma(a)=a$, consider $\beta>0$ arbitrarily fixed. By Proposition 4, there exists $b_{1} \in \mathbb{T} \backslash\{a\}$ such that

$$
\int_{\left[a, b_{1}\right)_{\mathbb{T}}} m_{\beta}(s) \Delta s \leq \beta .
$$

Let $\mathcal{A}$ the set of $\operatorname{arcs} x: \mathbb{T} \rightarrow \mathbb{R}^{n}$ satisfying

$$
\left\|x(t)-x_{0}\right\| \leq \beta
$$

for all $t \in\left[a, b_{1}\right]_{\mathbb{T}}$, and

$$
\left\|\int_{[c, d)_{\mathbb{T}}} x^{\Delta}(s) \Delta s\right\| \leq \int_{[c, d)_{\mathbb{T}}} m_{\beta}(s) \Delta s
$$

for each $c, d \in \mathbb{T}$ such that $c<d$.

Using the Proposition 3, we conclude that $\mathcal{A}$ is a compact set. It is easy to see also that $\mathcal{A}$ is a convex set.

For each $x \in \mathcal{A}$ define the function $g_{x}: \mathbb{T} \rightarrow \mathbb{R}^{n}$ by $g_{x}(s)=f\left(s, y_{x}(s)\right)$ for all $s \in \mathbb{T}$, where the function $y_{x}: \mathbb{T} \rightarrow \mathbb{R}^{n}$ is given by

$$
y_{x}(t)=\left\{\begin{array}{l}
x(t), t \in\left[a, b_{1}\right]_{\mathbb{T}} \\
x\left(b_{1}\right), \quad t \in \mathbb{T} \backslash\left[a, b_{1}\right]_{\mathbb{T}} .
\end{array}\right.
$$

Since $y_{x}$ is a continuous function, it follows from Lemma 9 that the function $g_{x}$ is $\Delta$-measurable.

Denote by $C\left(\mathbb{T}, \mathbb{R}^{n}\right)$ the set of all continuous functions with domain $\mathbb{T}$ and taking values on $\mathbb{R}^{n}$. We define the mapping $T: \mathcal{A} \rightarrow C\left(\mathbb{T}, \mathbb{R}^{n}\right)$ as

$$
(T x)(t)=x_{0}+\int_{[a, t)_{\mathbb{T}}} g_{x}(s) \Delta s
$$

for all $t \in \mathbb{T}$ and all $x \in \mathcal{A}$.

Consider $x \in \mathcal{A}$ arbitrarily fixed. From the Proposition 4, it follows that $T x$ is an arc. Now, it follows from Lemma 6 that

$$
(T x)^{\Delta}(t)=g_{x}(t) \quad \Delta \text {-a.e. } t \in[a, b)_{\mathbb{T}} .
$$

Hence, for each $c, d \in \mathbb{T}$ such that $c<d$ we have

$$
\begin{aligned}
\left\|\int_{[c, d)_{\mathbb{T}}}(T x)^{\Delta}(s) \Delta s\right\| & =\left\|\int_{[c, d)_{\mathbb{T}}} g_{x}(s) \Delta s\right\| \\
& \leq \int_{[c, d)_{\mathbb{T}}}\left\|g_{x}(s)\right\| \Delta s \leq \int_{[c, d)_{\mathbb{T}}} m_{\beta}(s) \Delta s .
\end{aligned}
$$

Finally, for each $t \in\left[a, b_{1}\right]_{\mathbb{T}}$ we deduce that

$$
\begin{aligned}
\left\|(T x)(t)-x_{0}\right\| & =\left\|\int_{[a, t)_{\mathbb{T}}} g_{x}(s) \Delta s\right\| \leq \int_{[a, t)_{\mathbb{T}}}\left\|g_{x}(s)\right\| \Delta s \\
& \leq \int_{[a, t)_{\mathbb{T}}} m_{\beta}(s) \Delta s \leq \int_{\left[a, b_{1}\right)_{\mathbb{T}}} m_{\beta}(s) \Delta s \leq \beta
\end{aligned}
$$

and then $T(\mathcal{A}) \subset \mathcal{A}$.

Take $\bar{x} \in \mathcal{A}$ arbitrary. Consider a sequence $\left\{x_{i}\right\}_{i \in \mathbb{N}} \subset \mathcal{A}$ such that

$$
\lim _{i \rightarrow \infty}\left\|x_{i}-\bar{x}\right\|_{\infty}=0 .
$$


For each $t \in \mathbb{T}$,

$$
\lim _{i \rightarrow \infty}\left\|g_{x_{i}}(t)-g_{\bar{x}}(t)\right\|=0
$$

and $\left\|g_{x_{i}}(t)-g_{\bar{x}}(t)\right\| \leq 2 m_{\beta}(t)$ for all $i \in \mathbb{N}$. It follows from the dominated convergence theorem [22] that

$$
\lim _{i \rightarrow \infty} \int_{[a, b)_{\mathbb{T}}}\left\|g_{x_{i}}(s)-g_{\bar{x}}(s)\right\| \Delta s=0
$$

and then

$$
\lim _{i \rightarrow \infty}\left\|T x_{i}-T \bar{x}\right\|_{\infty}=0
$$

Hence, $T$ is continuous in $\bar{x}$.

Since $C\left(\mathbb{T}, \mathbb{R}^{n}\right)$ equipped with the supremum norm is a Banach space, it follows from fixed point theorem of Schauder [25] that there exists $x_{*} \in \mathcal{A}$ such that $T x_{*}=x_{*}$. From Lemma 6, we may conclude that

$$
x_{*}^{\Delta}(t)=g_{x_{*}}(t)=f\left(t, x_{*}(t)\right) \quad \Delta \text {-a.e. } t \in\left[a, b_{1}\right)_{\mathbb{T}}
$$

and $x_{*}$ is a Carathéodory solution to Eq. (3).

\subsection{Euler solutions}

Let $\delta>0$ and $\pi=\left\{t_{0}, t_{1}, \ldots, t_{N}\right\} \in \mathcal{P}_{\delta}(\mathbb{T})$. Define the function $x_{\pi}: \mathbb{T} \rightarrow \mathbb{R}^{n}$ by

$$
\begin{array}{lll}
x_{\pi}\left(t_{0}\right)=x_{0}, & x_{\pi}(t)=x_{0}+\left(t-t_{0}\right) f\left(t_{0}, x_{0}\right) & t \in\left[t_{0}, t_{1}\right]_{\mathbb{T}} \\
x_{\pi}\left(t_{1}\right)=x_{1}, & x_{\pi}(t)=x_{1}+\left(t-t_{1}\right) f\left(t_{1}, x_{1}\right) & t \in\left[t_{1}, t_{2}\right]_{\mathbb{T}}
\end{array}
$$

and by induction

$$
x_{\pi}\left(t_{i}\right)=x_{i}, \quad x_{\pi}(t)=x_{i}+\left(t-t_{i}\right) f\left(t_{i}, x_{i}\right) \quad t \in\left[t_{i}, t_{i+1}\right]_{\mathbb{T}}
$$

as $i \in\{0,1, \ldots, N-1\}$. The arc $x_{\pi}$ is called the Euler polygonal arc.

An Euler solution to the problem stated in Eq. (2) is any $\operatorname{arc} x$ which is the uniform limit of a sequence of Euler polygonal $\operatorname{arcs} x_{\pi_{j}}$ related to partitions $\pi_{j} \in \mathcal{P}_{\delta_{j}}(\mathbb{T})$, for some sequence $\delta_{j} \downarrow 0$.

The existence of Euler solutions to Eq. (2) has been proven in [23] as follows.

Proposition 5 [23] Suppose that there exist $\gamma>0$ and $c_{*}>0$ such that

$$
\|f(t, x)\| \leq \gamma\|x\|+c_{*}
$$

for all $(t, x) \in \mathbb{T} \times \mathbb{R}^{n}$. Then the problem given by Eq. (2) has a Lipschitz-continuous Euler solution.

\subsection{Hermes solutions}

Below, we introduce and prove the existence of Hermes solutions to Eq. (2).

Definition 3 Let $x: \mathbb{T} \rightarrow \mathbb{R}^{n}$ be absolutely continuous. Then $x$ is called a Hermes solution (or $\mathcal{H}$-solution) to Eq. (2) if and only if there exist $\Delta$-measurable functions $p_{j}: \mathbb{T} \rightarrow \mathbb{R}^{n}$ and $\mathcal{C}$-solutions $x_{j}$ of

$$
\left\{\begin{array}{l}
y^{\Delta}(t)=f\left(t, y(t)+p_{j}(t)\right) \quad \Delta \text {-a.e. } t \in[a, b)_{\mathbb{T}} \\
y(a)=x_{0}
\end{array}\right.
$$


such that $p_{j} \rightrightarrows 0$ and $x_{j} \rightrightarrows x$.

We need the following technical result.

Lemma 10 [8] Consider the real numbers $r_{0}, r_{1}, \ldots, r_{N} \geq 0$ obeying

$$
r_{i+1} \leq\left(1+\beta_{i}\right) r_{i}+\eta_{i}, \quad i \in\{0,1, \ldots, N-1\}
$$

where $\beta_{i}, \eta_{i} \geq 0$ and $r_{0}=0$. Then

$$
r_{N} \leq\left(\exp \left(\sum_{i=0}^{N-1} \beta_{i}\right)\right) \sum_{i=0}^{N-1} \eta_{i}
$$

Let $\delta>0$ and take a partition $\alpha=\left\{t_{0}, t_{1}, \ldots, t_{N}\right\}$ in $\mathcal{P}_{\delta}(\mathbb{T})$. We denote by $x_{\alpha}$ the arc $x_{\alpha}: \mathbb{T} \rightarrow \mathbb{R}^{n}$ given by

$$
\begin{aligned}
& x_{\alpha}\left(t_{0}\right)=x_{0}, \quad x_{\alpha}(t)=x_{0}+\int_{\left[t_{0}, t\right)_{\mathbb{T}}} f\left(s, x_{0}\right) \Delta s \quad t \in\left[t_{0}, t_{1}\right]_{\mathbb{T}} \\
& x_{\alpha}\left(t_{1}\right)=x_{1}, \quad x_{\alpha}(t)=x_{1}+\int_{\left[t_{1}, t\right)_{\mathbb{T}}} f\left(s, x_{1}\right) \Delta s \quad t \in\left[t_{1}, t_{2}\right]_{\mathbb{T}}
\end{aligned}
$$

and by induction

$$
x_{\alpha}\left(t_{i}\right)=x_{i}, \quad x_{\alpha}(t)=x_{i}+\int_{\left[t_{i}, t\right)_{\mathbb{T}}} f\left(s, x_{i}\right) \Delta s \quad t \in\left[t_{i}, t_{i+1}\right]_{\mathbb{T}}
$$

as $i \in\{0,1, \ldots, N-1\}$. The arc $x_{\alpha}$ satisfies the following result.

Lemma 11 Suppose that $f$ is $\Delta$-measurable in $t$ and satisfies the condition given by Eq. (4). Then the arc $x_{\alpha}$ is such that

$$
\left\|x_{\alpha}^{\Delta}(t)\right\| \leq \gamma K+\gamma\left\|x_{0}\right\|+c_{*}:=L \quad \Delta \text {-a.e. } t \in[a, b)_{\mathbb{T}}
$$

where $K:=(b-a)\left(\gamma\left\|x_{0}\right\|+c_{*}\right) \exp (\gamma(b-a))$.

Proof From Lemma 6, we get

$$
x_{\alpha}^{\Delta}(t)=f\left(t, x_{i}\right) \quad \Delta \text {-a.e. } t \in\left[t_{i}, t_{i+1}\right) \mathbb{T}
$$

for all $i \in\{0,1, \ldots, N-1\}$. Since

$$
\left\|f\left(s, x_{i}\right)\right\| \leq \gamma\left\|x_{i}\right\|+c_{*} \leq \gamma\left\|x_{i}-x_{0}\right\|+\gamma\left\|x_{0}\right\|+c_{*}
$$

for each $i \in\{0,1, \ldots, N-1\}$ we have

$$
\begin{aligned}
\left\|x_{i+1}-x_{0}\right\| & \leq\left\|x_{i+1}-x_{i}\right\|+\left\|x_{i}-x_{0}\right\| \\
& =\left\|\int_{\left[t_{i}, t_{i+1}\right)_{\mathbb{T}}} f\left(s, x_{i}\right) \Delta s\right\|+\left\|x_{i}-x_{0}\right\| \\
& \leq \int_{\left[t_{i}, t_{i+1}\right)_{\mathbb{T}}}\left\|f\left(s, x_{i}\right)\right\| \Delta s+\left\|x_{i}-x_{0}\right\| \\
& \leq\left(t_{i+1}-t_{i}\right)\left\{\gamma\left\|x_{i}\right\|+c_{*}\right\}+\left\|x_{i}-x_{0}\right\| \\
& \leq\left(t_{i+1}-t_{i}\right)\left\{\gamma\left\|x_{i}-x_{0}\right\|+\gamma\left\|x_{0}\right\|+c_{*}\right\}+\left\|x_{i}-x_{0}\right\| \\
& =\left\{\left(t_{i+1}-t_{i}\right) \gamma+1\right\}\left\|x_{i}-x_{0}\right\|+\left(t_{i+1}-t_{i}\right)\left(\gamma\left\|x_{0}\right\|+c_{*}\right) .
\end{aligned}
$$


For each $i$ let $r_{i}=\left\|x_{i}-x_{0}\right\|, \beta_{i}=\left(t_{i+1}-t_{i}\right) \gamma$ and $\eta_{i}=\left(t_{i+1}-t_{i}\right)\left(\gamma\left\|x_{0}\right\|+c_{*}\right)$. Given $M \in\{1, \ldots, N\}$ we have

$$
r_{j+1} \leq\left(1+\beta_{j}\right) r_{j}+\eta_{j}
$$

for all $j \in\{0, \ldots, M-1\}$. Using Lemma 10 , we obtain

$$
\begin{aligned}
r_{M} & \leq\left[\exp \left(\beta_{0}+\beta_{1}+\cdots+\beta_{M-1}\right)\right]\left[\eta_{0}+\eta_{1}+\cdots+\eta_{M-1}\right] \\
& =\left[\exp \left(\left(t_{M}-t_{0}\right) \gamma\right)\right]\left[\left(t_{M}-t_{0}\right)\left(\gamma\left\|x_{0}\right\|+c_{*}\right)\right] \\
& \leq(b-a)\left(\gamma\left\|x_{0}\right\|+c_{*}\right) \exp (\gamma(b-a))=K .
\end{aligned}
$$

Thence

$$
\left\|x_{\alpha}^{\Delta}(t)\right\| \leq \gamma K+\gamma\left\|x_{0}\right\|+c_{*}:=L \quad \Delta \text {-a.e. } t \in[a, b)_{\mathbb{T}} .
$$

Theorem 6 (Existence for $\mathcal{H}$-solutions) Let $f$ be $\Delta$-measurable in $t$. Suppose, moreover, that $f$ satisfy the linear growth condition stated in Eq. (4). Then, there is a Lipschitz-continuous Hermes solution to Eq. (2).

Proof Let $\left\{\delta_{j}\right\}_{j \in \mathbb{N}} \subset \mathbb{R}$ be a sequence such that $\delta_{j} \downarrow 0$. For each $j$ take a partition $\alpha_{j}=\left\{t_{0}^{(j)}, t_{1}^{(j)}, \ldots, t_{N(j)}^{(j)}\right\}$ in $\mathcal{P}_{\delta_{j}}(\mathbb{T})$ and let $x_{\alpha_{j}}$ be the arc $x_{\alpha_{j}}: \mathbb{T} \rightarrow \mathbb{R}^{n}$ given by

$$
\begin{aligned}
& x_{\alpha_{j}}\left(t_{0}^{(j)}\right)=x_{0}, x_{\alpha_{j}}(t)=x_{0}+\int_{\left[t_{0}^{(j)}, t\right)_{\mathbb{T}}} f\left(s, x_{0}\right) \Delta s t \in\left[t_{0}^{(j)}, t_{1}^{(j)}\right]_{\mathbb{T}} \\
& x_{\alpha_{j}}\left(t_{1}^{(j)}\right)=x_{1}^{(j)}, x_{\alpha_{j}}(t)=x_{1}^{(j)}+\int_{\left[t_{1}^{(j)}, t\right)_{\mathbb{T}}} f\left(s, x_{1}^{(j)}\right) \Delta s t \in\left[t_{1}^{(j)}, t_{2}^{(j)}\right]_{\mathbb{T}}
\end{aligned}
$$

and by induction

$$
x_{\alpha_{j}}\left(t_{i}^{(j)}\right)=x_{i}^{(j)}, \quad x_{\alpha_{j}}(t)=x_{i}^{(j)}+\int_{\left[t_{i}^{(j)}, t\right)_{\mathbb{T}}} f\left(s, x_{i}^{(j)}\right) \Delta s \quad t \in\left[t_{i}^{(j)}, t_{i+1}^{(j)}\right]_{\mathbb{T}}
$$

as $i \in\{0,1, \ldots, N(j)-1\}$. Without loss of generality we relabel $\left\{x_{\alpha_{j}}\right\}$ by $\left\{x_{j}\right\}$.

Because of Lemma 11, there exists $L>0$ such that

$$
\left\|x_{j}^{\Delta}(t)\right\| \leq L \quad \Delta \text {-a.e. } t \in[a, b)_{\mathbb{T}}
$$

for all $j$. Since $x_{j}(a)=x_{0}$, using Proposition 3, there exists a subsequence $\left\{x_{j_{k}}\right\} \subset\left\{x_{j}\right\}$ and an $\operatorname{arc} x: \mathbb{T} \rightarrow \mathbb{R}^{n}$ such that $x_{j_{k}} \rightrightarrows x$.

Since $x_{j}$ is a Lipschitz continuous function with constant $L$ for each $j$, then $x$ is a Lipschitz continuous function with constant $L$.

For each $j$ define the function $p_{j}: \mathbb{T} \rightarrow \mathbb{R}^{n}$ as

$$
p_{j}(t)=x_{i}^{(j)}-x_{j}(t)
$$

for each $t \in\left[t_{i}^{(j)}, t_{i+1}^{(j)}\right)_{\mathbb{T}}$ and $p_{j}(b)=0$. Consider $t \in\left[t_{i}^{(j)}, t_{i+1}^{(j)}\right)_{\mathbb{T}}$ arbitrarily fixed. If $\rho\left(t_{i+1}^{(j)}\right)=t_{i}^{(j)}$ we have $t=t_{i}^{(j)}$ and then $p_{j}(t)=0$. If $t_{i+1}^{(j)}-t_{i}^{(j)} \leq \delta_{j}$ it follows that $\left\|p_{j}(t)\right\|=\left\|x_{i}^{(j)}-x_{j}(t)\right\| \leq L\left|t-t_{i}^{(j)}\right| \leq L \delta_{j}$ and so $p_{j} \rightrightarrows 0$.

From Lemma 6, we get

$$
x_{j}^{\Delta}(t)=f\left(t, x_{i}^{(j)}\right)=f\left(t, x_{j}(t)+p_{j}(t)\right) \quad \Delta \text {-a.e. } t \in\left[t_{i}^{(j)}, t_{i+1}^{(j)}\right)_{\mathbb{T}}
$$


and then

$$
x_{j}^{\Delta}(t)=f\left(t, x_{j}(t)+p_{j}(t)\right) \quad \Delta \text {-a.e. } t \in[a, b)_{\mathbb{T}} .
$$

Therefore $x$ is a Hermes solution to Eq. (2).

\subsection{Krasovskii solutions}

The set-valued mapping $K f: \mathbb{T} \times \mathbb{R}^{n} \rightsquigarrow \mathbb{R}^{n}$ is defined by

$$
K f(t, x)=\bigcap_{\varepsilon>0} \overline{c o} f(t, x+\varepsilon B)
$$

where $x+\varepsilon B$ represents the open ball of radius $\varepsilon$ about the point $x$.

Definition 4 An $\operatorname{arc} x: \mathbb{T} \rightarrow \mathbb{R}^{n}$ is a Krasovskii solution (or $\mathcal{K}$-solution) to Eq. (2) if it satisfies the dynamic inclusion

$$
x^{\Delta}(t) \in K f(t, x(t)) \quad \Delta \text {-a.e. } t \in[a, b)_{\mathbb{T}}
$$

and $x(a)=x_{0}$.

If $f$ satisfies the condition stated in Eq. (4), then

$$
K f(t, x) \subset\left(\gamma\|x\|+c_{*}\right) \bar{B}
$$

for all $(t, x) \in \mathbb{T} \times \mathbb{R}^{n}$. Hence, $K f(t, x)$ is a nonempty compact convex subset of $\mathbb{R}^{n}$ for all $(t, x) \in \mathbb{T} \times \mathbb{R}^{n}$.

The graph of $K f$ is defined by the set

$$
G r K f:=\left\{(t, x, \gamma) \in \mathbb{T} \times \mathbb{R}^{n} \times \mathbb{R}^{n}: \gamma \in K f(t, x)\right\} .
$$

We have the following Lemma concerning the graph of $K f$.

Lemma 12 The set-valued mapping $K f$ has closed graph.

Proof Let $(t, x, \gamma) \in \overline{G r K f}$. Thus, there exists a sequence $\left\{\left(t_{i}, x_{i}, \gamma_{i}\right)\right\}_{i \in \mathbb{N}} \subset G r K f$ such that

$$
\lim _{i \rightarrow \infty}\left(t_{i}, x_{i}, \gamma_{i}\right)=(t, x, \gamma)
$$

Take $\varepsilon>0$ arbitrary. Then there exists $i_{0} \in \mathbb{N}$ such that for $i \geq i_{0}, x_{i} \in x+\varepsilon B$. If $\varepsilon_{i}=\left\|x_{i}-x\right\|$ if follows that $\gamma_{i} \in \overline{c o} f\left(t, x_{i}+\varepsilon_{i} B\right) \subset \overline{c o} f(t, x+2 \varepsilon B)$, for all $i \geq i_{0}$. Hence, $\gamma \in K f(t, x)$ and, therefore, $K f$ has closed graph.

From [23, Theorem 7], we get the existence for Krasovskii solutions.

Proposition 6 (Existence for $\mathcal{K}$-solutions) Let $f$ obey the condition given by Eq. (4). Then, there is a Krasovskii solution to Eq. (2).

From the definition of $K f$, it is clear that $f(t, x) \in K f(t, x)$ for all $(t, x) \in \mathbb{T} \times \mathbb{R}^{n}$. Thence $\mathcal{C} \subset \mathcal{K}$, that is, every Carathéodory solution is a Krasovskii solution. 


\subsubsection{Closure theorem for Krasovskii solutions}

In the following theorem, we obtain an extension of the closure theorem for Krasovskii solutions [15] to systems of dynamic equations on time scales.

Theorem 7 (Closure theorem for $\mathcal{K}$-solutions) Let $f: \mathbb{T} \times \mathbb{R}^{n} \rightarrow \mathbb{R}^{n}$ be locally bounded. Let $\left\{x_{j}\right\}_{j \in \mathbb{N}}$ be a sequence of arcs $x_{j}: \mathbb{T} \rightarrow \mathbb{R}^{n}$ such that for each $j$, we have

$$
x_{j}^{\Delta}(t) \in K f\left(t, x_{j}(t)\right) \quad \Delta \text {-a.e. } t \in[a, b)_{\mathbb{T}}
$$

and $x_{j}(a)=x_{0}$. If $\left\{x_{j}\right\}$ converges uniformly to the function $x: \mathbb{T} \rightarrow \mathbb{R}^{n}$, then $x$ is a Krasovskii solution to Eq. (2).

Proof Fix an arbitrary number $\epsilon>0$. Then there exists $j_{0} \in \mathbb{N}$ such that

$$
\left\|x_{j}(t)-x(t)\right\| \leq\left\|x_{j}-x\right\|_{\infty}<\epsilon
$$

for all $t \in \mathbb{T}$ and all $j \geq j_{0}$. Hence, for each $j \geq j_{0}$ we have

$$
x_{j}^{\Delta}(t) \in \overline{c o} f\left(t, x_{j}(t)+\epsilon B\right) \subset \overline{c o} f(t, x(t)+2 \epsilon B) \quad \Delta \text {-a.e. } t \in[a, b)_{\mathbb{T}} .
$$

Since $\|x(t)\| \leq \epsilon+\left\|x_{j_{0}}\right\|_{\infty}$ for each $t \in \mathbb{T}$ and $f$ is locally bounded, there exists $r>0$ satisfying

$$
\overline{c o} f(t, x(t)+2 \epsilon B) \subset r \bar{B}
$$

for all $t \in \mathbb{T}$. Thus,

$$
\left\|x_{j}^{\Delta}(t)\right\| \leq r \quad \Delta \text {-a.e. } t \in[a, b)_{\mathbb{T}}
$$

for all $j \geq j_{0}$.

For each $j \geq j_{0}$ we also have $\left\|x_{j}(a)\right\| \leq \epsilon+\|x(a)\|$. From Proposition 3, it follows that there is a subsequence $\left\{x_{j_{k}}\right\} \subset\left\{x_{j}\right\}$ and an arc $z: \mathbb{T} \rightarrow \mathbb{R}^{n}$ such that $x_{j_{k}} \rightrightarrows z$. Since $x_{j_{k}} \rightrightarrows x$, we conclude that $x \equiv z$.

If $t \in[a, b)_{\mathbb{T}}$ and $\sigma(t)>t$, we have

$$
x_{j}^{\Delta}(t)=\frac{x_{j}(\sigma(t))-x_{j}(t)}{\mu(t)} \in \overline{c o} f(t, x(t)+2 \epsilon B)
$$

and then

$$
\lim _{j \rightarrow \infty} x_{j}^{\Delta}(t)=x^{\Delta}(t) \in \overline{c o} f(t, x(t)+2 \epsilon B) .
$$

Let $f_{j}: \mathbb{T} \rightarrow \mathbb{R}^{n}$ be defined by $f_{j}(t)=x_{j}^{\Delta}(t)$. Then, $f_{j} \in L_{1}\left([a, b)_{\mathbb{T}}, \mathbb{R}^{n}\right)$ and so $\tilde{f}_{j} \in L_{1}\left([a, b), \mathbb{R}^{n}\right)$.

Define the set-valued mapping $\Gamma: \mathbb{T} \rightsquigarrow \mathbb{R}^{n}$ by

$$
\Gamma(t)=\overline{c o} f(t, x(t)+2 \epsilon B) .
$$

Also consider the set-valued mapping $\tilde{\Gamma}:[a, b] \rightsquigarrow \mathbb{R}^{n}$ given by

$$
\tilde{\Gamma}(t)=\left\{\begin{array}{l}
\Gamma(t), t \in \mathbb{T} \\
\Gamma\left(t_{i}\right), \quad t \in\left(t_{i}, \sigma\left(t_{i}\right)\right) \quad \text { for some } i \in I .
\end{array}\right.
$$

For each $i \in I$, we have

$$
\tilde{f}_{j}(t)=f_{j}\left(t_{i}\right)=x_{j}^{\Delta}\left(t_{i}\right) \in \Gamma\left(t_{i}\right)=\tilde{\Gamma}(t)
$$


for all $t \in\left[t_{i}, \sigma\left(t_{i}\right)\right)$.

If

$$
A:=\left\{t \in[a, b)_{\mathbb{T}}: \sigma(t)=t\right\}
$$

and

$$
C=\left\{t \in[a, b)_{\mathbb{T}}: f_{j}(t) \notin \Gamma(t)\right\}
$$

we have $D \subset A \cap C$, where

$$
D=\left\{t \in[a, b): \tilde{f}_{j}(t) \notin \tilde{\Gamma}(t)\right\} .
$$

Hence,

$$
\lambda^{*}(D) \leq \lambda^{*}(A \cap C)=m^{*}(A \cap C) \leq m^{*}(C)=0
$$

and then

$$
\tilde{f}_{j}(t) \in \tilde{\Gamma}(t) \text { a.e. } t \in[a, b) .
$$

Since $\tilde{\Gamma}(t) \subset r \bar{B}$, we obtain

$$
\left\|\tilde{f}_{j}(t)\right\| \leq r \text { a.e. } t \in[a, b) .
$$

Define the arc $y_{j}:[a, b] \rightarrow \mathbb{R}^{n}$ by

$$
y_{j}(t)=x_{j}(a)+\int_{[a, t)} \tilde{f}_{j}(s) d s .
$$

If $t \in \mathbb{T}$,

$$
y_{j}(t)=x_{j}(a)+\int_{[a, t)_{\mathbb{T}}} f_{j}(s) \Delta s=x_{j}(a)+\int_{[a, t)_{\mathbb{T}}} x_{j}^{\Delta}(s) \Delta s=x_{j}(t) .
$$

Since $\left\{y_{j}(a)\right\}$ is a bounded sequence and

$$
\dot{y}_{j}(t)=\tilde{f}_{j}(t) \text { a.e. } t \in[a, b)
$$

it follows from Proposition 3 that there is a subsequence $\left\{y_{j_{k}}\right\} \subset\left\{y_{j}\right\}$ and an arc $y:[a, b] \rightarrow$ $\mathbb{R}^{n}$ such that $y_{j_{k}} \rightrightarrows y$.

If $t \in \mathbb{T}$ we have

$$
\lim _{k \rightarrow \infty} y_{j_{k}}(t)=x(t)
$$

and so $y(t)=x(t)$.

For each $s<t$ in $[a, b]$,

$$
y_{j_{k}}(t)-y_{j_{k}}(s)=\int_{[s, t)} \tilde{f_{j_{k}}}(\tau) d \tau=\int_{[s, t]} \tilde{f}_{j_{k}}(\tau) d \tau \in \int_{[s, t]} \tilde{\Gamma}(\tau) d \tau .
$$

Using Lemma 7, we obtain

$$
y(t)-y(s) \in \int_{[s, t]} \tilde{\Gamma}(\tau) d \tau
$$

and by Lemma 8 ,

$$
\dot{y}(\tau) \in \overline{c o} \tilde{\Gamma}(\tau)=\tilde{\Gamma}(\tau) \text { a.e. } \tau \in[a, b) .
$$


Let $t \in A$ such that there exist the derivatives $x^{\Delta}(t)$ and $\dot{y}(t)$. Consider the sequence $\left\{s_{i}\right\}_{i \in \mathbb{N}} \subset \mathbb{T}$ such that $s_{i} \downarrow t$. Hence,

$$
\dot{y}(t)=\lim _{i \rightarrow \infty} \frac{y\left(s_{i}\right)-y(t)}{s_{i}-t}=\lim _{i \rightarrow \infty} \frac{x\left(s_{i}\right)-x(t)}{s_{i}-t}=x^{\Delta}(t)
$$

and then $H=\left\{t \in[a, b)_{\mathbb{T}}: x^{\Delta}(t) \notin \Gamma(t)\right\} \subset A \cap(E \cup F)$, where

$$
E=\{t \in[a, b): \nexists \dot{y}(t)\}
$$

and

$$
F=\{t \in[a, b): \dot{y}(t) \notin \tilde{\Gamma}(t)\} .
$$

We have

$$
\begin{aligned}
m^{*}(H) & \leq m^{*}(A \cap(E \cup F))=\lambda^{*}(A \cap(E \cup F)) \\
& \leq \lambda^{*}(E \cup F) \leq \lambda^{*}(E)+\lambda^{*}(F)=0
\end{aligned}
$$

and, therefore,

$$
x^{\Delta}(t) \in \overline{c o} f(t, x(t)+2 \epsilon B) \quad \Delta \text {-a.e. } t \in[a, b)_{\mathbb{T}} .
$$

Take a sequence $\left\{\epsilon_{i}\right\}_{i \in \mathbb{N}} \subset \mathbb{R}$ such that $\epsilon_{i} \downarrow 0$. If the set $Z$ is given by

$$
Z=\left\{t \in[a, b)_{\mathbb{T}}: x^{\Delta}(t) \notin K f(t, x(t))\right\}
$$

it follows that

$$
Z \subset \bigcup_{i \in \mathbb{N}} W_{i}
$$

where

$$
W_{i}=\left\{t \in[a, b)_{\mathbb{T}}: x^{\Delta}(t) \notin \overline{c o} f\left(t, x(t)+\epsilon_{i} B\right)\right\} .
$$

Since $m^{*}\left(W_{i}\right)=0$, using Lemma 4, we may conclude that $m^{*}(Z)=0$ and then

$$
x^{\Delta}(t) \in K f(t, x(t)) \quad \Delta \text {-a.e. } t \in[a, b)_{\mathbb{T}}
$$

proving the theorem.

The proof of the result below is similar to proof of Theorem 7 .

Proposition 7 Suppose that $f: \mathbb{T} \times \mathbb{R}^{n} \rightarrow \mathbb{R}^{n}$ is locally bounded. If an arc $x: \mathbb{T} \rightarrow \mathbb{R}^{n}$ is a $\mathcal{H}$-solution to Eq. (2) then $x$ is a $\mathcal{K}$-solution to Eq. (2).

However, the relationship $\mathcal{K} \subset \mathcal{H}$ for autonomous systems in continuous time [15, Theorem 5.5], it is not valid for the time scale $\mathbb{T}=\{a, b\}$. Indeed, if $x_{0}=0$ and $f:\{a, b\} \times \mathbb{R} \rightarrow \mathbb{R}$ is given by

$$
f(t, x)= \begin{cases}1, & x \geq 0 \\ -1, & x<0\end{cases}
$$

it follows that $x=0$ is a Krasovskii solution but not a Hermes solution. If $x=0$ was a Hermes solution, there would $\Delta$-measurable functions $p_{j}:\{a, b\} \rightarrow \mathbb{R}$ and $\mathcal{C}$-solutions $x_{j}$ of

$$
\left\{\begin{array}{l}
y^{\Delta}(t)=f\left(t, y(t)+p_{j}(t)\right) \quad \Delta \text {-a.e. } t \in[a, b)_{\mathbb{T}} \\
y(a)=0
\end{array}\right.
$$


such that $p_{j} \rightrightarrows 0$ and $x_{j} \rightrightarrows 0$. Since $[a, b)_{\mathbb{T}}=\{a\}$ we have

$$
x_{j}^{\Delta}(a)=\frac{x_{j}(b)-x_{j}(a)}{b-a}=f\left(a, x_{j}(a)+p_{j}(a)\right)
$$

and taking $j \rightarrow \infty$ we obtain a contradiction.

\subsection{Filippov solutions}

We define the set-valued mapping $F f: \mathbb{T} \times \mathbb{R}^{n} \rightsquigarrow \mathbb{R}^{n}$ by

$$
F f(t, x)=\bigcap_{\varepsilon>0} \bigcap_{Z ; \lambda(Z)=0} \overline{c o} f(t,(x+\varepsilon B) \backslash Z)
$$

where $\lambda$ denotes the Lebesgue measure.

Definition 5 Let $x: \mathbb{T} \rightarrow \mathbb{R}^{n}$ be an arc. Then $x$ is called a Filippov solution (or $\mathcal{F}$-solution) to Eq. (2) if and only if

$$
x^{\Delta}(t) \in F f(t, x(t)) \quad \Delta \text {-a.e. } t \in[a, b)_{\mathbb{T}}
$$

and $x(a)=x_{0}$.

Suppose that $f$ satisfies the condition given by Eq. (4). Hence,

$$
F f(t, x) \subset\left(\gamma\|x\|+c_{*}\right) \bar{B}
$$

for all $(t, x) \in \mathbb{T} \times \mathbb{R}^{n}$. Thus, $F f(t, x)$ is a nonempty compact convex subset of $\mathbb{R}^{n}$ for all $(t, x) \in \mathbb{T} \times \mathbb{R}^{n}$.

Also it holds the following result concerning the set-valued mapping $F f$.

Lemma 13 The graph of $F f$ is closed.

Proof Take $(t, x, \gamma) \in \overline{G r F f}$. Then there exists a sequence $\left\{\left(t_{i}, x_{i}, \gamma_{i}\right)\right\}_{i \in \mathbb{N}} \subset G r F f$ such that

$$
\lim _{i \rightarrow \infty}\left(t_{i}, x_{i}, \gamma_{i}\right)=(t, x, \gamma)
$$

Fix an arbitrary $\varepsilon>0$ and a subset $Z \subset \mathbb{R}^{n}$ satisfying $\lambda(Z)=0$. Hence, there exists $i_{0} \in \mathbb{N}$ such that $x_{i} \in x+\varepsilon B$ for $i \geq i_{0}$. Let $\varepsilon_{i}=\left\|x_{i}-x\right\|$ so $\gamma_{i} \in \overline{c o} f\left(t,\left(x_{i}+\varepsilon_{i} B\right) \backslash Z\right) \subset$ $\overline{c o} f(t,(x+2 \varepsilon B) \backslash Z)$, for all $i \geq i_{0}$. Then $\gamma \in F f(t, x)$ and we conclude that $F f$ has closed graph.

Thus, using [23, Theorem 7], we obtain the existence for Filippov solutions.

Proposition 8 (Existence for $\mathcal{F}$-solutions) Suppose that $f$ satisfies the linear growth condition stated in Eq. (4). Then, there is a Filippov solution to Eq. (2).

We have $F f(t, x) \subset K f(t, x)$ for all $(t, x) \in \mathbb{T} \times \mathbb{R}^{n}$. Thus, $\mathcal{F} \subset \mathcal{K}$ and so every Filippov solution is a Krasovskii solution.

In the next theorem, we extend to systems of dynamic equations on time scales the result [15, Lemma 2.8], concerning autonomous systems. 
Lemma 14 Let $f$ be such that $f(t, x)=g(x)$ for all $(t, x) \in \mathbb{T} \times \mathbb{R}^{n}$. Suppose that there exists a disjoint decomposition

$$
\mathbb{R}^{n}=\bigcup M_{i} \text { with } M_{i} \subset \overline{\operatorname{Int} M_{i}}
$$

and continuous $f_{i}: \mathbb{R}^{n} \rightarrow \mathbb{R}^{n}$ such that $g=f_{i}$ on $M_{i}$. Then each $\mathcal{K}$-solution is a $\mathcal{F}$-solution $($ so $\mathcal{F}=\mathcal{K}$ ).

Proof From the proof of [15, Lemma 2.8], we have $K g(x) \subset F g(x)$ for all $x \in \mathbb{R}^{n}$. Since $K g(x)=K f(t, x)$ and $F g(x)=F f(t, x)$ for each $(t, x) \in \mathbb{T} \times \mathbb{R}^{n}$, we conclude that $K f(t, x) \subset F f(t, x)$ for all $(t, x) \in \mathbb{T} \times \mathbb{R}^{n}$. Therefore, $\mathcal{F}=\mathcal{K}$.

We also have that the relationship $\mathcal{F} \subset \mathcal{H}$ for autonomous systems in continuous time [17, Lemma 3], does not extend to the time scale $\mathbb{T}=\{a, b\}$. To see this, we need just consider $x_{0}=0$ and the function $f:\{a, b\} \times \mathbb{R} \rightarrow \mathbb{R}$ given by

$$
f(t, x)= \begin{cases}1, & x \geq 0 \\ -1, & x<0 .\end{cases}
$$

Thus, $x=0$ is a Filippov solution but not a Hermes solution.

\subsection{Comparison between Carathéodory and Filippov solutions}

In the two examples below, we verified that there is not a direct relationship between Carathéodory and Filippov solutions whenever we consider two discontinuous vector fields to the dynamic equation given by Eq. (2).

Example 1 Let $f: \mathbb{T} \times \mathbb{R} \rightarrow \mathbb{R}$ be defined as

$$
f(t, x)= \begin{cases}1, & x=0 \\ 0, & x \neq 0\end{cases}
$$

and suppose that $x_{0}=0$. Since $F f(t, 0)=\{0\}, x=0$ is a Filippov solution but not a Carathéodory solution.

Example 2 Consider $f: \mathbb{T} \times \mathbb{R} \rightarrow \mathbb{R}$ defined by

$$
f(t, x)= \begin{cases}0, & x=0 \\ 1, & x \neq 0\end{cases}
$$

and let $x_{0}=0$. Then $x=0$ is a Carathéodory solution but not a Filippov solution.

On the other hand, if the vector field $f: \mathbb{T} \times \mathbb{R}^{n} \rightarrow \mathbb{R}^{n}$ is continuous with respect to $x$, the set $F f(t, x)$ coincides with $f(t, x)$, as stated in [11].

So, if the function $f$ is continuous with respect to $x$, we have $\mathcal{C}=\mathcal{F}=\mathcal{K}$.

\subsection{Comparison between Euler and the other generalized solutions}

Comparisons between Euler and other generalized solutions to discontinuous differential equations can be found in [1]. Below, we provide some comparisons between Euler and other generalized solutions for the time scale $\mathbb{T}=\{a, b\}$.

Given a function $f:\{a, b\} \times \mathbb{R}^{n} \rightarrow \mathbb{R}^{n}$, the arc $x:\{a, b\} \rightarrow \mathbb{R}^{n}$ defined by $x(a)=x_{0}$ and $x(b)=x_{0}+(b-a) f\left(a, x_{0}\right)$ is the only Euler solution. From the proof of Theorem 6 , we also have that the $\operatorname{arc} x$ is a Hermes solution. Hence, $\mathcal{E} \subset \mathcal{H} \subset \mathcal{K}$.

In the following examples, we see that the relation $\mathcal{E}=\mathcal{H}=\mathcal{K}$ for autonomous systems in continuous time [1, Corollary 4.5], does not hold for the time scale $\mathbb{T}=\{a, b\}$. 
Example 3 Consider $f:\{a, b\} \times \mathbb{R} \rightarrow \mathbb{R}$ given by

$$
f(t, x)= \begin{cases}1, & x=0 \\ -1, & x \neq 0\end{cases}
$$

and let $x_{0}=0$. Hence, $x=0$ is a Krasovskii solution but not an Euler solution.

Example 4 Let $f:\{a, b\} \times \mathbb{R} \rightarrow \mathbb{R}$ be defined by

$$
f(t, x)= \begin{cases}0, & x=0 \\ \frac{1}{b-a}, & x \neq 0\end{cases}
$$

and let $x_{0}=0$. Thus, the arc $x:\{a, b\} \rightarrow \mathbb{R}$ defined by $x(a)=x_{0}$ and $x(b)=1$ is a Hermes solution but not an Euler solution.

Below, we also consider comparisons between Euler and Filippov solutions.

Example 5 If $f:\{a, b\} \times \mathbb{R} \rightarrow \mathbb{R}$ is given by

$$
f(t, x)= \begin{cases}1, & x=0 \\ 0, & x \neq 0\end{cases}
$$

and $x_{0}=0$, then $x=0$ is a Filippov solution but not an Euler solution.

Example 6 Let $x_{0}=0$ and define $f:\{a, b\} \times \mathbb{R} \rightarrow \mathbb{R}$ by

$$
f(t, x)= \begin{cases}0, & x=0 \\ 1, & x \neq 0 .\end{cases}
$$

Thus, $x=0$ is an Euler solution but not a Filippov solution.

\section{References}

1. al Shammari, K.: Filippov's operator and discontinuous differential equations. ProQuest LLC, Ann Arbor, MI. Ph.D. Thesis, Louisiana State University and Agricultural and Mechanical College (2006)

2. Bohner, M., Peterson, A.: Dynamic Equations on Time Scales. Birkhäuser, Boston (2001). An introduction with applications

3. Bohner, M., Peterson, A.: First and second order linear dynamic equations on time scales. J. Differ. Equ. Appl. 7(6), 767-792 (2001)

4. Cabada, A., Vivero, D.R.: Criterions for absolute continuity on time scales. J. Differ. Equ. Appl. 11(11), 1013-1028 (2005)

5. Cabada, A., Vivero, D.R.: Expression of the Lebesgue $\Delta$-integral on time scales as a usual Lebesgue integral: application to the calculus of $\Delta$-antiderivatives. Math. Comput. Model. 43(1-2), 194-207 (2006)

6. Ceragioli, F.M.: Discontinuous ordinary differential equations and stabilization. Ph.D. Thesis, Università degli Studi di Firenze (1999)

7. Cichoń, M., Kubiaczyk, I., Sikorska-Nowak, A., Yantir, A.: Existence of solutions of the dynamic Cauchy problem in Banach spaces. Demonstr. Math. 45(3), 561-573 (2012)

8. Clarke, F.H., Ledyaev, Y.S., Stern, R.J., Wolenski, P.R.: Nonsmooth Analysis and Control Theory, Graduate Texts in Mathematics, vol. 178. Springer, New York (1998)

9. Coddington, E.A., Levinson, N.: Theory of Ordinary Differential Equations. McGraw-Hill Book Company Inc, New York (1955)

10. Dai, Q., Tisdell, C.C.: Existence of solutions to first-order dynamic boundary value problems. Int. J. Differ. Equ. 1(1), 1-17 (2006)

11. Filippov, A.F.: Differential equations with discontinuous right-hand side. Mat. Sb. (N.S.) 51(93), 99-128 (1960)

12. Gilbert, H.: Existence theorems for first-order equations on time scales with $\Delta$-Carathéodory functions. Adv. Differ. Equ. 2010, 20 (2010). Article ID 650827 
13. Guseinov, G.S.: Integration on time scales. J. Math. Anal. Appl. 285(1), 107-127 (2003)

14. Guseinov, G.S., Kaymakçalan, B.: Basics of Riemann delta and nabla integration on time scales. J. Differ. Equ. Appl. 8(11), 1001-1017 (2002)

15. Hájek, O.: Discontinuous differential equations. I. J. Differ. Equ. 32(2), 149-170 (1979)

16. Hale, J.K.: Ordinary Differential Equations, 2nd edn. Robert E. Krieger Publishing Co., Inc., Huntington (1980)

17. Hermes, H.: Discontinuous vector fields and feedback control. In: Differential Equations and Dynamical Systems (Proc. Internat. Sympos., Mayaguez, P. R., 1965), pp. 155-165. Academic Press, New York (1967)

18. Krasovskiı̆, N.N.: Igrovye zadachi o vstreche dvizhenii. Izdat. Nauka, Moscow (1970)

19. Loewen, P.D.: Optimal control via nonsmooth analysis. CRM Proceedings and Lecture Notes, vol. 2. American Mathematical Society, Providence (1993)

20. Peterson, A.C., Tisdell, C.C.: Boundedness and uniqueness of solutions to dynamic equations on time scales. J. Differ. Equ. Appl. 10(13-15), 1295-1306 (2004)

21. Royden, H.L.: Real Analysis. The Macmillan Co., New York (1963)

22. Rudin, W.: Real and Complex Analysis, 3rd edn. McGraw-Hill Book Co., New York (1987)

23. Santos, I.L.D., Silva, G.N.: Absolute continuity and existence of solutions to dynamic inclusions in time scales. Math. Ann. 356(1), 373-399 (2013)

24. Santos, I.L.D., Silva, G.N.: Filippov's selection theorem and the existence of solutions for optimal control problems in time scales. Comput. Appl. Math. 33(1), 223-241 (2014)

25. Schauder, J.: Der Fixpunktsatz in Funktionalräumen. Stud. Math. 2, 171-180 (1930)

26. Tisdell, C.C., Zaidi, A.: Basic qualitative and quantitative results for solutions to nonlinear, dynamic equations on time scales with an application to economic modelling. Nonlinear Anal. 68(11), 3504-3524 (2008) 